\title{
19 low mass hyper-velocity star candidates from the first data release of LAMOST survey
}

\author{
Yin-bi Li ${ }^{1}$, A-Li Luo* ${ }^{*}$, Gang Zhao*1, You-jun $\mathrm{Lu}^{1}$, Peng Wei ${ }^{1,2}$, Bing $\mathrm{Du}^{1}$, Xiang $\mathrm{Li}^{1}$, \\ Yong-Heng Zhao ${ }^{1}$, Zhan-wen $\mathrm{Han}^{3}$, Bo Wang ${ }^{3}$, Yue $\mathrm{Wu}^{1}$, Yong Zhang ${ }^{4}$, Yong-hui $\mathrm{Hou}^{4}$, Yue-fei \\ Wang $^{4}$ and Ming Yang ${ }^{1}$ \\ ${ }^{1}$ Key Laboratory of Optical Astronomy, National Astronomical Observatories, Chinese Academy of \\ Sciences, Beijing 100012, China; lal@nao.cas.cn,gzhao@bao.ac.cn \\ ${ }^{2}$ State Key Laboratory of High-end Server \& Storage Technology, Jinan 250510, China \\ ${ }^{3}$ Key Laboratory for the Structure and Evolution of Celestial Objects, Yunnan Observatories, Chinese \\ Academy of Sciences, Kunming, 650216, China \\ ${ }^{4}$ Nanjing Institute of Astronomical Optics \& Technology, National Astronomical Observatories, Chinese \\ Academy of Sciences, Nanjing 210042, China
}

\begin{abstract}
Hyper-velocity stars are believed to be ejected out from the Galactic center through dynamical interactions between (binary) stars and the central massive black hole(s). In this paper, we report 19 low mass F/G/K type hyper-velocity star candidates from over one million stars of the first data release of the LAMOST general survey. We determine the unbound probability for each candidate using a Monte-Carlo simulation by assuming a non-Gaussian proper-motion error distribution, Gaussian heliocentric distance and radial velocity error distributions. The simulation results show that all the candidates have unbound possibilities over $50 \%$ as expected, and one of them may even exceed escape velocity with over $90 \%$ probability. In addition, we compare the metallicities of our candidates with the metallicity distribution functions of the Galactic bulge, disk, halo and globular cluster, and conclude that the Galactic bulge or disk is likely the birth place for our candidates.
\end{abstract}

Key words: stars: low-mass — stars: kinematics and dynamics — Galaxy: abundances stars: fundamental parameters — stars: distances

\section{INTRODUCTION}

The hypervelocity stars (HVSs), discovered in the Galactic halo, are travelling so fast that they can escape from the Galaxy. Hills (1988) first predicted their existence, and the existence are the powerful evidence of massive black hole at the center of the Milky Way. A natural explanation is that they may be ejected

* Corresponding Author. 
out from the Galactic center (GC) by interactions of stars with the massive black hole (MBH) or the hypothetical binary MBHs as predicted by Hills (1988) and Yu \& Tremaine (2003). Such ejection mechanisms can be divided into three categories: tidal breakup of binary stars in the vicinity of a single $\mathrm{MBH}$ (Hills 1988; Yu \& Tremaine 2003; Bromley et al. 2006), and the binary stars are probably injected into the vicinity of the MBH from the young stellar disk in the GC (e.g., Lu et al. 2010; Zhang et al. 2010) or from the Galactic bulge (Perets 2009a b) ; single star encounters with a binary MBH (Yu \& Tremaine 2003; Sesana et al.|2007; Merritt|2006); or single star encounters with a cluster of stellar mass black holes around the MBH (O’Leary \& Loeb 2008).

However, the black hole acceleration mechanism cannot explain a type of HVSs such as US 708 (Hirsch et al. 2005), which is not originated from the center of our Galaxy (e.g., Geier et al. 2015). This type of HVSs is likely to be the ejected donor remnant of a thermonuclear supernova in the white dwarf + helium star scenario (see Wang \& Han 2009; Geier et al. 2015), in which LAMOST are searching for such type of HVSs based on the theoretical results of Wang \& Han (2009).

Alternatively, other ejection models can also accelerate stars to high speed. For example, binary disruption in the dense stellar clusters such as Galactic disk (Blaauw 1961; Leonard \& Dewey 1993; Napiwotzki \& Silva 2012), in such case, a supernova explosion of more massive evolved component can accelerate its companion to high speed. Tidal disruptions of dwarf galaxies in the Milky Way can also produce high velocity stars (Abadi et al. 2009; Lu et al. 2010; Teyssier et al. 2009), such mechanism can produce high speed star stream or a population of old isolated 'escaped' (unbound) or 'wondering' (bound) stars.

Seventeen years after Hill's prediction, three HVSs were successively discovered (Brown et al. 2005; Hirsch et al. 2005; Edelmann et al. 2005), they are massive O or B type stars located in the Galactic halo. Until recently, over 20 unbound HVS were identified (Brown et al. 2009, 2012; Zheng et al. 2014), most of them are massive B type stars, and are located at distant Galactic halo with Galactocentric distances larger than $25 \mathrm{kpc}$. An interesting exception is a identified B type HVS discovered by Zheng et al. (2014), which is the first HVS discovered in the Large Sky Area Multi-Object Fiber Spectroscopic Telescope (LAMOST) survey, and it is the brightest HVS currently known, and locate at a Galatocentric distance of $13 \mathrm{kpc}$.

Assuming a Salpeter initial mass function (IMF), the expected solar mass HVSs are about 10 times more abundant than the 3-4 M $\odot$ HVSs (Brown et al. 2009). Kollmeier et al. (2009) systematically searched for such low mass HVSs in about 290,000 spectra of the Sloan Digital Sky Survey (SDSS) (York et al. 2000; Gunn et al. 1998, 2006), however, they found only 6 metal-poor stars that can be possibly taken as HVS candidates. Li et al. (2012) also searched F and G type HVSs from over 370,000 stellar spectra of data release seven of the SDSS, and they presented a low mass metal-poor HVS candidate catalogue. Palladino et al. (2014) identified $20 \mathrm{G}$ and K type HVS candidates from approximately 240,000 stars of the Sloan Extension for Galactic Understanding and Exploration (SEGUE) (Yanny et al. 2009) G and K dwarf samples. Besides, Zhong et al. (2014) reported a catalog of 28 high velocity star candidates from the first data release (DR1) of the LAMOST general survey (Luo et al. 2015), which cover a much broader color range than ever, and 17 of them are F, G, or K type low mass stars. These current searching results might suggest that the IMF of the parent population of these HVSs is top heavy. A top heavy IMF of the HVS 
parent population is possibly consistent with the disk origination (Bartko et al. 2010; Zhang et al. 2013; Kollmeier et al. 2010). In order to distinguish the ejection mechanisms of HVSs and put constraints on the origin of the parent population of HVSs, it is quite necessary to search for the low-mass HVSs.

In this paper, we systematically search and investigate HVSs with stellar spectra of LAMOST DR1, and totally find 19 HVS candidates. The structure of this paper is as follows. In Section 2, we introduce the LAMOST and DR1 data productions in detail. In Section 3, we present a series of spectroscopic, photometric and dynamic criteria to select HVS candidates. In Section 4, we analyze the probability for each HVS candidate that they can escape from our Galaxy. In Section 5, we compare the metallicities of our 19 HVS candidates with the $[\mathrm{Fe} / \mathrm{H}]$ distributions of Galactic bulge, disk, halo and globular cluster, and conclude that our HVS candidates are likely originated from the Galactic bulge or disk. Finally, the discussion and conclusion are given in Section 6.

\section{THE FIRST DATA RELEASE OF THE LAMOST GENERAL SURVEY}

The LAMOST is a 4 meter quasi-meridian reflecting Schmidt telescope, it adopts novel active optics technique, which allows both a large effective aperture of about $4 \mathrm{~m}$ and a wide field of view of $5^{\circ}$. The focal surface of LAMOST has 4000 precisely positioned optical fibers, which are equally connected to 16 spectrographs, thus it can observe 4000 targets simultaneously. Each spectrograph is equipped with a red channel CCD camera and a blue one, which can simultaneously provide red and blue spectra of observed targets respectively (Cui et al. 2012; Wang et al. 1996; Su \& Cui 2004).

The primary scientific goal of LAMOST survey is to investigate the large-scale structure of the universe, as well as structure and evolution history of Galaxy, and it consists of two main parts. The first part is the LAMOST Extra-Galactic Survey (LEGAS) of galaxies, and the second part is the LAMOST Experiment for Galactic Understanding and Exploration (LEGUE) Survey of the Milky Way (Zhao et al. 2012; Deng et al. 2012). Considering the science goals and the targets available, the LEGAS is consists of galaxy survey and QSO survey, and the LEGUE is divided into three parts, i.e., the Galactic anticenter survey, the disk survey, and the spheroid survey (Liu et al. 2014; Chen et al. 2012; Carlin et al. 2012). The Galactic anticenter survey (LSS-GAC) covers a significant volume of the Galactic thin/thick disks and halo in a continuous sky area of $\sim 3,400$ square degree, which is centered on Galactic anti-center area with Galactic longitudes $150^{\circ} \leq 1 \leq 210^{\circ}$ and latitudes $|b| \leq 30^{\circ}$ (Liu et al. 2014; Yuan et al. 2014). The disk survey choose eight low and bright plates along the Galactic plane which are nearly uniformed distributed in the region $0^{\circ}<$ $\alpha<67^{\circ}$ and $42^{\circ}<\delta<59^{\circ}$ (Chen et al. 2012). The halo survey mainly focuses on areas of SDSS survey, which plan to observe 5.8 million objects with $r<16.8$ at $|b|>30^{\circ}$ (Yang et al. 2012; Deng et al. 2012; Zhang et al. 2012).

After one year pilot survey (Luo et al. 2012), LAMOST began its first year general survey from September 2012, and completed its first data release (DR1) to domestic data users and foreign collaborators in June 2013. DR1 totally published 2,204,696 wavelength-calibrated and relative flux-calibrated spectra, which are consists of 1,944,329 stars, 12,082 galaxies, 5,017 quasars, and 243,268 unknown objects. These spectra cover a wavelength range of 3690-9100 $\AA$ with a resolution of $\mathrm{R} \sim 1800$. In addition, DR1 also published five spectroscopic parameter catalogs, which are the General catalog, the A, F, G and K 


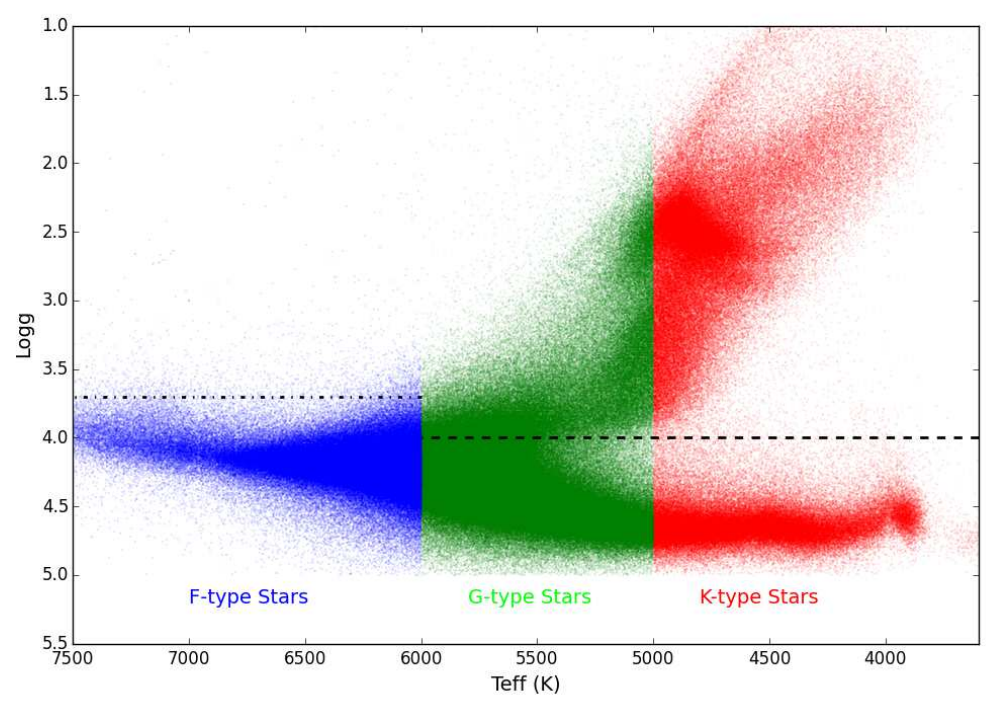

Fig. 1 The Hertzsprung-Russell diagram of F, G and K type stars of LAMOST DR1, where blue, green and red points represent F, G, and K type stars respectively. The black dot-dash line shows the location of $\log g=3.75$ for $\mathrm{F}$ type stars, and the black dashed line displays $\log g=4.0$ for $\mathrm{G}$ and $\mathrm{K}$ type stars.

type stars catalog, the A type stars catalog, the $\mathrm{M}$ dwarfs catalog and the observed plate information catalog respectively. The A, F, G and $\mathrm{K}$ type stars catalog provides effective temperatures (Teff), surface gravities $(\log g)$, metallicities $([\mathrm{Fe} / \mathrm{H}])$ and heliocentric radial velocities for 1,061,918 stars, these spectroscopic parameters are indispensable for searching and studying hyper-velocity stars (Luo et al. 2015).

\section{IDENTIFICATION OF THE LAMOST DR1 HVS CANDIDATES}

Our HVS candidates are drawn from F, G and K dwarfs of the LAMOST DR1, we use five steps to search for them, and the numbers in below brackets indicate the number of stars left after each step.

1. Selecting F, G and K dwarfs from LAMOST DR1[519,027]. The LAMOST DR1 officially released atmospheric parameters for 1,061,918 A, F, G and K type stars, which are derived by the LAMOST Stellar Parameter Pipeline (LASP) (Luo et al. 2015). We fist select F, G and K type stars with $3600 \leq$ Teff $\leq 7500$, and plot their Hertzsprung-Russell diagram in Figure 1. From Figure 1, we can see that F, G and K dwarfs can be initially selected with the criteria: 1) $\log g>4.0$, when $3600 \leq$ Teff $<6000$; 2) $\log g>3.75$, when $6000 \leq$ Teff $\leq 7500$, and we totally obtain 737,023 F, G and K type dwarfs. Then, we upload equatorial coordinates of our 737,023 dwarfs to the 'MyDB' database of SDSS DR10, and obtain photometry information of 521,618 dwarfs from the 'PhotoObjall' table, as well as proper motions of 519,027 dwarfs from the 'ProperMotions' table. Finally, 519,027 F, G and K dwarfs with both photometries and proper motions are selected. 
2. Further selecting F, G and K dwarfs with the color and magnitude methods[191,405].

(a) $g_{0}<20.2, r_{0}<19.7,0.2<(g-r)_{0}<0.48$, for F dwarfs

$r_{0}<19.7,0.48<(g-r)_{0}<0.55$, for $\mathrm{G}$ dwarfs

$r_{0}<19.0,0.55<(g-r)_{0}<0.75$, for K dwarfs

(b) $A(\mathrm{r})<0.5 \mathrm{mag}$

(c) $|b| \geq 10$

(d) $p s f$ MagErr_g $/ r / i<0.05$ mag, mode $=1$ and clean $=1$

(e) $0.2 \mathrm{mag}<(g-i)_{0}<4.0 \mathrm{mag}$

Newby et al. (2011) investigated the photometric uncertainties of SDSS, and pointed out that they are constant up to r-band apparent magnitude $\left(r_{0}\right)$ of 19.7 , thus we use above criterion (a) to select $F, G$ and K dwarfs. Schlesinger et al. (2012) mentioned that above and below the Galactic plane undergo small amounts of extinction, this small amount of reddening can affect target selection, so we use criteria (b) to retain F, G, and K dwarfs samples with extinction in r-band $(\mathrm{A}(\mathrm{r}))$ less than 0.5 mag $(\mathrm{A}(\mathrm{r})<0.5$ mag). As we known that Schlegel et al. (1998) map has a limited spatial resolution and fails at low Galactic latitudes, extinction from the Schlegel et al. (1998) map may not represent the true value of targets which may lead to inaccurate distance estimates, thus we use criterion (c) to remove dwarfs with $|b|<10$. In addition, in order to make sure SDSS photometry reliable, we use criterion (d) to constrain SDSS psf mag errors in $\mathrm{g}, \mathrm{r}$, and i band lower than $0.05 \mathrm{mag}$, and the value of photometric flag 'mode' and 'clean' are 1. In the end, we constrain our dwarf samples to the color range of $0.2<(g-i)_{0}<4.0$ mag, which is the reliable range to estimate absolute magnitude with the photometric parallax relation of Ivezić et al. (2008).

3. Calculate phase space coordinates and escape velocities[191,405]. We first calculate heliocentric distances for 191,405 F, G, and K dwarfs using the distance modulus:

$$
d(\mathrm{kpc})=\frac{10^{0.2 \times\left(\mathrm{r}_{0}-\mathrm{M}_{\mathrm{r}}\right)}}{100}
$$

where $d$ is the heliocentric distance in unit of kpc, $r_{0}$ is the r-band deredden apparent magnitude directly from the SDSS DR10 'PhotoObjAll' table, and $M_{r}$ is the r-band absolute magnitude.

The above step constrains our dwarfs in the color range $0.2<(g-i)_{0}<4.0 \mathrm{mag}$, thus we use the photometric parallax relation proposed by Ivezić et al. (2008) to estimate $M_{r}$ :

$$
M_{r}\left((g-i)_{0},[F e / H]\right)=M_{r}^{0}\left((g-i)_{0}\right)+\Delta M_{r}([F e / H])
$$

where $M_{r}^{0}(g-i)$ is the color-magnitude relation, $\Delta M_{r}([\mathrm{Fe} / \mathrm{H}])$ is the absolute magnitude correction, and they can be calculated by the equations (A7) and (A2) of Ivezić et al. (2008), respectively. The $(g-i)_{0}$ color can be available from the table 'PhotoObjAll' of SDSS DR10, and the metal abundance $[\mathrm{Fe} / \mathrm{H}]$ can be obtained from the A, F, G and $\mathrm{K}$ type stars catalog of LAMOST DR1.

Then, we use a right-handed cartesian coordinate system centered on the GC to calculate Galactic three dimension (3D) space positions, the $\mathrm{X}$ axis pointing from the Sun to the Galactic center (GC) with the Sun at $x=-8 \mathrm{kpc}$, the $\mathrm{Y}$ axis pointing in the direction of rotation and the $\mathrm{Z}$ axis pointing towards the Northern Galactic Pole. We use a similar coordinate system to calculate Galactic 3D velocity, and 
assume that the motion of the local standard of rest (LSR) is $220 \mathrm{~km} \mathrm{~s}^{-1}$, and the velocity of the Sun with respect to the LSR is $\left(11.1 \mathrm{~km} \mathrm{~s}^{-1}, 12.24 \mathrm{~km} \mathrm{~s}^{-1}, 7.25 \mathrm{~km} \mathrm{~s}^{-1}\right.$ ) (Schönrich et al. 2010).

To determine which dwarfs are unbound to the Galaxy, we adopt five different Galactic potential models to estimate escape velocities $\left(V_{\text {esc }}\right)$, i.e., a spherically symmetric convergent model Xue et al. 2008, hereafter Xue08), a spherically symmetric divergent model (Kenyon et al. 2008, hereafter Kenyon08), two axisymmetric divergent models (Paczynski 1990; Koposov et al. 2010, hereafter Paczynski90 and Koposov10), and a triaxial convergent model (Gnedin et al. 2005, hereafter Gnedin05). Beside a simple model of Kenyon08, other four potentials are all three-component bulge-disk-halo models. Xue08, Koposov10 and Gnedin05 adopt a spherical bulge (Hernquist 1990), which is different from the Miyamoto-Nagai bulge (Miyamoto \& Nagai 1975) of Paczynski90. Paczynski90, Koposov10, and Gnedin05 use the Miyamoto-Nagai disk(Miyamoto \& Nagai 1975), while Xue08 adopt an exponential disk. For the halo component, the four three component models are entirely different Navarro et al. 1996; Paczynski 1990; Fellhauer et al. 2006). For the convergent Galactic potential model, there exists a true escape velocity, and we use equation 3 to estimate $V_{\text {esc }}$ :

$$
\left|v_{\mathrm{esc}}(r)\right|=\sqrt{2 \times|\Phi(r)|}
$$

However, for the divergent potential, any HVS with finite space velocity could not really escape from the Galaxy. We thus define unbound stars as ones which can reach $r \geq 200 \mathrm{kpc}$ with $v \geq+200 \mathrm{~km} \mathrm{~s}^{-1}$ as shown in equation 4 (Kenyon et al. 2008):

$$
\left|v_{\mathrm{esc}}(r)\right|=\sqrt{2 \times\left(\frac{1}{2} \times 200^{2}+|| \Phi(r)|-| \Phi(200) \|\right)}
$$

4. Find HVS candidates with clean or reliable proper motions[32]. In step two, we use a series of criteria to make sure that our dwarf samples have reliable SDSS photometric parameters, which affect heliocentric distance estimate accuracy. In addition, the proper motion distribution of SDSS tends to display large proper motion errors, we thus need to ensure that proper motions of dwarf samples are real rather than the product of large errors.

Munn et al. (2004, 2008) presented an improved proper-motion catalog, which matched each SDSS point source to the USNO-B catalog (Monet et al. 2003). The SDSS+USNO-B catalog is $90 \%$ complete

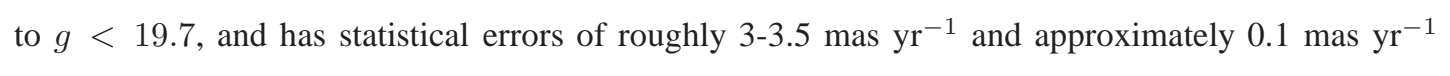
systematic errors for each component proper-motion. Munn et al. (2004) also defined a series of criteria to make sure that the proper-motions from SDSS catalog are reliable, these criteria were later revised by Kilic et al. (2006) for their white dwarf samples, and Palladino et al. (2014) used the revised criteria when they search for G and K type HVS candidates from the SEGUE. In this paper, we use the criteria to select HVS candidates with 'clean' or 'reliable' proper-motions, the 'clean' proper-motion is defined as follows:

- match $=1$, which represents only a USNO-B object is within 1" radius of the SDSS target.

- sigRA $<525$ and sigDEC $<525$, which means that the proper-motion fit must have rms residuals less than 525 mas in both right ascension and declination directions.

- nFit $\geq 6$, which shows at least six observations (SDSS+USNO-B) have been used to determine the proper motion. 
- dist22 $>7$, which suggests the distance to the nearest neighbor with $g<22$ must exceed 7". and the 'reliable' proper-motion is defined as follows:

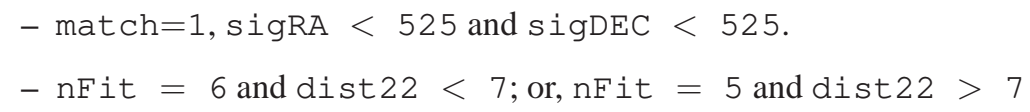

Kilic et al. (2006) pointed out the contamination rate for a target with a 'reliable' proper motion is not larger than $1.5 \%$. Finally, we totally find 32 HVS candidates which can escape from the Galaxy in at least one Galactic potential model mentioned in step three, 15 of them have 'clean' proper motions, and other 17 candidates have 'reliable' proper motions.

5. Finally, HVS candidates are selected with high quality spectra and reliable atmospheric parameters[19]. After above four steps, 32 HVS candidates are initially selected from over 190,000 F, G and K dwarf samples, we further visually inspect their LAMOST spectra, and find ten proper-motion 'clean' candidates and nine proper-motion 'reliable' candidates, which have high quality spectra, and their $\mathrm{r}$ band signal-to-noise ratio 'SNR_r' are listed in Table 1 Lee et al. (2008) presents the SEGUE Stellar Parameter Pipeline (SSPP), which was used to derive the fundamental stellar atmospheric parameters (Teff, logg, and [Fe/H]) for A,F,G and $\mathrm{K}$ type stars using multiple methods. We use the version of the SSPP used for the seventh data release of the SDSS to verify atmospheric parameters for 19 HVS candidates, and conclude that the parameters derived by the SSPP are roughly consistent with those obtained by the LASP.

Here, we finally select 19 HVS candidates, all of them have reliable photometry and stellar atmospheric parameters, over $98.5 \%$ probability of robust proper-motions, high quality spectra and no visual blending. Their fundamental parameters such as equatorial coordinates, r-band dereddened apparent magnitudes and atmospheric parameters, are shown in Table1, and their heliocentric distances, Galactic distances, Galactic total velocities, and escape velocities obtained by five Galactic potential models are listed in Table 2.

From Table 1, we can see that the value of $[\mathrm{Fe} / \mathrm{H}]$ errors obtained from the LAMOST parameter catalog seem to be large, even much larger than their true value, such large errors will effect the distance error estimates. From Table 2 we can see that the Galactic distance errors are really large, and a fraction of them can reach up to or over 10\%. Luo et al. (2015) pointed out that external errors released in the LAMOST parameters catalog are larger than the real measurement errors, because they rescaled the external errors using a ratio. They compared the LASP parameters with high resolution spectra results, SDSS DR9 results, and results from Gao et al. (2015) and LSP3(Xiang et al. 2015), the mean external error is 0.125 dex which is much close to the true error. Considering this mean error as $[\mathrm{Fe} / \mathrm{H}]$ errors of our 19 candidates, the corresponding Galactic distance errors listed in the brackets of the third column in Table 2 are really much smaller.

Theoretically, Ivezić et al.(2008) presented out that a $1.0 \mathrm{dex}[\mathrm{Fe} / \mathrm{H}]$ error will bring about a $1 \mathrm{mag}$ absolute magnitude $(\mathrm{Mr})$ error at the median thin-disk metallicity $([\mathrm{Fe} / \mathrm{H}]=-0.2)$, and a $0.57 \mathrm{mag} \mathrm{Mr}$ error at the median halo metallicity $([\mathrm{Fe} / \mathrm{H}]=-1.50)$, which represents the mean LAMOST external $[\mathrm{Fe} / \mathrm{H}]$ error of 0.125 dex will result in a Mr error of 0.125 mag at most, and an approximately $6 \%$ Galactic distance error. 
Besides, we check whether our candidates are new findings. Kollmeier et al. (2009) present 6 F/G type metal-poor HVS candidates from over 290,000 SDSS stars, Li et al. (2012) proposed $13 \mathrm{~F}$ and G type metalpoor unbound HVS candidates from SDSS DR7, and Palladino et al. (2014) found $20 \mathrm{G}$ and K type unbound HVS candidates in SEGUE G and K dwarf samples from the SDSS DR9. Our 19 HVS candidates are not in the three HVS catalogs by checking equatorial coordinates. Besides, Zhong et al. (2014) presented a catalog of 28 high-velocity star candidates from LAMOST DR1, they use a velocity criterion $|r v|>200 \mathrm{~km} \mathrm{~s}^{-1}$ and $\left|V_{\mathrm{gt}}\right| \geq 300 \mathrm{~km} \mathrm{~s}^{-1}$ when selecting HVS candidates, where $r v$ is the heliocentric radial velocity, and $V_{\mathrm{gt}}$ is the 3D total velocity, and this criterion prevent our candidates except one to be found. Because the exceptional candidate is not in the $u-g$ and $r-i$ color ranges where Zhong et al. (2014) estimate accurate photometric metallicity, it is also included in our HVS candidates catalog. Among their 28 HVS samples, there are 12 stars with spectra type earlier than F type, and other 16 candidates are eliminated by our photometry and proper-motion criteria. In summary, we can conclude that our 19 low mass F/G/K type HVS candidates are new findings.

\section{ESTIMATING THE RELIABILITY OF OUR CANDIDATES WITH MONTE-CARLO METHOD}

Although we use a series of criteria to ensure our HVS candidates with reliable photometry, atmospheric parameters and proper-motions in section 3, it is still premature to say that the final HVS samples do not contain false-positive detections. With this in mind, we thus consider the probability that our HVS candidates are in fact unbound to the Milky Way. To obtain such an unbound probability for each HVS sample, we built a Monte-Carlo simulation to sample a million realizations of orbit parameters.

Dong et al. (2011) present a non-Gaussian probability distribution function (PDF) of proper-motion errors using quasar samples with 'clean' proper-motions, which contains a Gaussian core and an extended wing. Applying this error distribution model, we randomly produce a million total proper-motion errors $\left(p m_{\text {error }}\right)$ with the inverse function method. Assuming the proper-motion error is isotropic in the ra-dec plane, we can produce a million angles ' $\theta$ ' using an uniform distribution model, and obtain a million proper-motion errors in the ra and dec direction using $p m r a_{\text {error }}=p m_{\text {error }} \times \cos (\theta)$ and $p m d e c_{\text {error }}=p m_{\text {error }} \times \sin (\theta)$, where $p m r a_{\text {error }}$ and $p m d e c_{\text {error }}$ are random proper-motion errors in ra and dec directions respectively. Using the proper-motion measurements from SDSS in ra and dec directions, two component proper-motion errors from SDSS and the random proper-motion errors $p m r a_{\mathrm{error}}$ and pmdec $_{\text {error, }}$ we can obtain a million random two components proper-motions.

In addition, we randomly generate a million radial velocities and heliocentric distances assuming a Gaussian error distribution function, and a million random Galactic 6D phase space coordinates and escape velocities at each Galactic distance in the million realization can be further obtained. In such a Monte-Carlo simulation, unbound probability for each HVS candidate can be derived by the fraction $\frac{N_{V_{\mathrm{gt}}}>V_{\mathrm{esc}}}{1000000}$, where

$V_{\text {gt }}$ and $V_{\text {esc }}$ are Galactic total space velocities and escape velocities respectively, and $N_{V_{\mathrm{gt}}}>V_{\mathrm{esc}}$ is the number of $V_{\mathrm{gt}}$ larger than $V_{\mathrm{esc}}$ in a million realizations.

Table 3 lists unbound possibilities for each HVS candidate, and '_-' in this table represents that the candidate is not unbound in this potential model, and we do not calculate unbound possibilities in these cases. 
Table 1 Fundamental parameters of 19 HVS candidates.

\begin{tabular}{|c|c|c|c|c|c|c|c|c|c|c|c|c|}
\hline HVS & Designation & $\begin{array}{c}\mathrm{ra}^{a} \\
(\text { degree })\end{array}$ & $\begin{array}{c}\operatorname{dec}^{a} \\
\text { (degree) }\end{array}$ & $\begin{array}{c}\mathrm{r}^{b} \\
(\mathrm{mag})\end{array}$ & $\mathrm{SNR} \_\mathbf{r}^{c}$ & $\begin{array}{c}\mathrm{rv}_{\odot}{ }^{d} \\
\left(\mathrm{~km} \mathrm{~s}^{-1}\right)\end{array}$ & $\begin{array}{c}\mu_{\alpha} \cos (\delta)^{e} \\
\left(\mathrm{mas} \mathrm{yr}^{-1}\right)\end{array}$ & $\begin{array}{c}\mu_{\delta}{ }^{e} \\
\left(\operatorname{mas~yr}^{-1}\right)\end{array}$ & $\begin{array}{l}\operatorname{Teff}^{f} \\
(\mathrm{~K})\end{array}$ & $\operatorname{logg}^{f}$ & {$[\mathrm{Fe} / \mathrm{H}]^{f}$} & {$[\mathrm{Mg} / \mathrm{Fe}]^{g}$} \\
\hline 1 & J172524.12+565709.6 & 261.3505 & 56.95267 & 13.95 & 101 & $-103 \pm 11$ & $-17.19 \pm 2.62$ & $88.11 \pm 2.62$ & $5251 \pm 102$ & $4.65 \pm 0.43$ & $-0.76 \pm 0.36$ & 0.43 \\
\hline 2 & $\mathrm{~J} 170333.23+373102.3$ & 255.8885 & 37.51733 & 17.50 & 29 & $-60 \pm 10$ & $-22.60 \pm 3.00$ & $18.79 \pm 3.00$ & $5069 \pm 156$ & $4.40 \pm 0.41$ & $0.08 \pm 0.38$ & -0.05 \\
\hline 3 & $\mathrm{~J} 132422.30+312841.6$ & 201.0929 & 31.47823 & 15.76 & 18 & $-23 \pm 16$ & $-24.35 \pm 2.36$ & $-27.53 \pm 2.36$ & $5828 \pm 330$ & $4.03 \pm 0.66$ & $0.02 \pm 0.62$ & 0.33 \\
\hline 4 & J091255.48+140413.8 & 138.2312 & 14.07052 & 15.26 & 13 & $46 \pm 22$ & $9.82 \pm 2.52$ & $-54.49 \pm 2.52$ & $6231 \pm 307$ & $4.67 \pm 0.36$ & $-0.86 \pm 0.82$ & 0.57 \\
\hline 6 & J133115.50+150438.9 & 202.8146 & 15.07748 & 18.49 & 62 & $-29 \pm 6$ & $-20.87 \pm 3.05$ & $4.08 \pm 3.05$ & $4854 \pm 90$ & $4.68 \pm 0.29$ & $0.12 \pm 0.24$ & -0.03 \\
\hline 7 & J175513.55+511927.4 & 268.8065 & 51.3243 & 13.87 & 38 & $-72 \pm 11$ & $8.06 \pm 2.73$ & $48.98 \pm 2.73$ & $5228 \pm 170$ & $4.45 \pm 0.47$ & $-0.34 \pm 0.46$ & 0.32 \\
\hline 8 & $\mathrm{~J} 113116.03+571131.1$ & 172.8168 & 57.19199 & 16.19 & 23 & $-91 \pm 11$ & $-43.52 \pm 2.64$ & $-38.49 \pm 2.64$ & $6083 \pm 259$ & $4.07 \pm 0.50$ & $-1.47 \pm 0.89$ & 0.58 \\
\hline 9 & J121811.06+284659.9 & 184.5461 & 28.78333 & 14.08 & 54 & $-18 \pm 8$ & $-48.94 \pm 2.57$ & $-0.32 \pm 2.57$ & $5068 \pm 117$ & $4.64 \pm 0.35$ & $0.04 \pm 0.30$ & -0.03 \\
\hline 13 & J063934.38+280912.8 & 99.89327 & 28.15358 & 17.02 & 8 & $15 \pm 30$ & $0.11 \pm 2.52$ & $15.04 \pm 2.52$ & $6064 \pm 431$ & $3.94 \pm 0.59$ & $-0.33 \pm 0.88$ & -0.18 \\
\hline 14 & $\mathrm{~J} 005233.53+413322.6$ & 13.13972 & 41.5563 & 14.81 & 23 & $-30 \pm 23$ & $-48.83 \pm 2.55$ & $-32.97 \pm 2.55$ & $6187 \pm 282$ & $4.34 \pm 0.48$ & $-0.43 \pm 0.64$ & 0.02 \\
\hline 15 & J012947.93-021343.2 & 22.44971 & -2.228684 & 14.85 & 50 & $13 \pm 13$ & $-24.22 \pm 3.82$ & $30.44 \pm 3.82$ & $5860 \pm 183$ & $4.25 \pm 0.56$ & $-0.36 \pm 0.42$ & 0.26 \\
\hline 16 & $\mathrm{~J} 075303.30+272657.0$ & 118.2638 & 27.44918 & 17.81 & 14 & $75 \pm 32$ & $-18.13 \pm 3.00$ & $4.51 \pm 3.00$ & $6017 \pm 399$ & $4.26 \pm 0.70$ & $-0.39 \pm 0.75$ & 0.36 \\
\hline 17 & $\mathrm{~J} 142235.20+455631.3$ & 215.6467 & 45.94204 & 14.89 & 44 & $-121 \pm 12$ & $-42.77 \pm 2.43$ & $11.14 \pm 2.43$ & $5231 \pm 138$ & $4.68 \pm 0.43$ & $-0.57 \pm 0.44$ & 0.38 \\
\hline 18 & J130744.34-004449.5 & 196.9348 & -0.747102 & 17.18 & 9 & $14 \pm 20$ & $-9.72 \pm 3.11$ & $11.48 \pm 3.11$ & $6035 \pm 408$ & $4.28 \pm 0.50$ & $-0.27 \pm 0.86$ & 0.56 \\
\hline 19 & $\mathrm{~J} 103858.44+565558.1$ & 159.7435 & 56.93283 & 13.74 & 74 & $-15 \pm 14$ & $-88.10 \pm 5.61$ & $97.36 \pm 5.61$ & $5785 \pm 146$ & $4.28 \pm 0.54$ & $-0.74 \pm 0.41$ & 0.36 \\
\hline
\end{tabular}

Notes. The candidates HVS1-HVS10 all have 'clean' proper-motions, and the candidates HVS11-

HVS19 have 'reliable' proper-motions.

${ }^{a}$ Equatorial coordinate from the SDSS 'PhotoObjAll' catalog.

${ }^{b}$ Dereddened $\mathrm{r}$ band apparent magnitude from the SDSS 'PhotoObjAll' catalog.

${ }^{c}$ r-band signal to noise ratio from LAMOST parameter catalog.

${ }^{d}$ Heliocentric radial velocity from LAMOST parameter catalog.

${ }^{e}$ Proper motion in both right ascension and declination directions from the SDSS 'ProperMotions' catalog

${ }^{f}$ Atmospheric parameters from the LAMOST parameter catalog.

${ }^{g}$ Note that there are large uncertainties in $[\mathrm{Mg} / \mathrm{Fe}]$ measurements.

From this table, we can see that unbound probability for each HVS candidate exceeds 0.5 as expected, and it is even over 0.9 for HVS1 in the Xue08 potential model. In addition, for each HVS candidate, the value of unbound probability depends on the adopted Galaxy potential model to a certain extent, such as HVS1, the probability varies from 0.64 in the Gnedin 05 model to 0.93 in the Xue08 model, and the probability difference between two potential models changes from 0.07 to 0.29. Among our 19 HVS samples, only seven candidates are unbound in all five potential models, Figure 2 shows their distribution of $V_{\text {gal }}-V_{\text {esc }}$, where $V_{\text {gal }}$ and $V_{\text {esc }}$ are total Galactic velocities and escape velocities of each realization. From this figure, we can see that the total velocity exceeds escape velocity in most cases.

Actually, the value of unbound probability mainly relies on the difference between total space velocity and escape velocity. When the total velocity is much larger than the escape velocity, the effect of parameter error and Galactic potential model will be extremely small. Conversely, when total velocity is just larger than the escape velocity, parameter error and potential model will greatly affect the unbound probability. 
Table 2 Kinematic parameters of 19 HVS candidates.

\begin{tabular}{|c|c|c|c|c|c|c|c|c|}
\hline HVS & $\begin{array}{l}\mathrm{d}_{\odot}{ }^{a} \\
(\mathrm{kpc})\end{array}$ & $\begin{array}{l}\mathrm{R}_{\mathrm{G}}{ }^{b} \\
(\mathrm{kpc})\end{array}$ & $\begin{array}{c}\mathrm{V}_{\mathrm{G}^{c}} \\
\left(\mathrm{~km} \mathrm{~s}^{-1}\right)\end{array}$ & $\begin{array}{c}\mathrm{V}_{\mathrm{esc}}-\mathrm{Xue}^{d} \\
\left(\mathrm{~km} \mathrm{~s}^{-1}\right)\end{array}$ & $\begin{array}{c}\mathrm{V}_{\mathrm{esc}}-\text { Pacyznski }^{d} \\
\left(\mathrm{~km} \mathrm{~s}^{-1}\right)\end{array}$ & $\begin{array}{c}\mathrm{V}_{\mathrm{esc}}-\mathrm{Koposv}^{d} \\
\left(\mathrm{~km} \mathrm{~s}^{-1}\right)\end{array}$ & $\begin{array}{c}\mathrm{V}_{\text {esc }}-\text { Kenyon }^{d} \\
\left(\mathrm{~km} \mathrm{~s}^{-1}\right)\end{array}$ & $\begin{array}{c}\mathrm{V}_{\text {esc }}-\text { Gnedin }^{d} \\
\left(\mathrm{~km} \mathrm{~s}^{-1}\right) \\
\end{array}$ \\
\hline 1 & $1.5 \pm 0.2$ & $8.0 \pm 0.2(0.1)$ & $644 \pm 96$ & 491 & 540 & 573 & 592 & 607 \\
\hline 2 & $4.6 \pm 0.9$ & $7.5 \pm 0.9(0.3)$ & $626 \pm 98$ & 500 & 540 & 572 & 603 & 608 \\
\hline 3 & $4.6 \pm 1.5$ & $9.0 \pm 1.5(0.5)$ & $572 \pm 195$ & 493 & 523 & 555 & 595 & 596 \\
\hline 4 & $2.9 \pm 0.9$ & $10.1 \pm 0.9(0.3)$ & $563 \pm 178$ & 474 & 519 & 551 & 574 & 590 \\
\hline 5 & $3.7 \pm 1.4$ & $8.7 \pm 1.4(0.4)$ & $540 \pm 218$ & 492 & 527 & 559 & 593 & 599 \\
\hline 6 & $5.9 \pm 0.8$ & $8.7 \pm 0.8(0.5)$ & $524 \pm 101$ & 505 & 524 & 555 & 608 & 598 \\
\hline 7 & $1.9 \pm 0.4$ & $7.9 \pm 0.4(0.2)$ & $503 \pm 83$ & 492 & 541 & 575 & 594 & 608 \\
\hline 8 & $2.5 \pm 0.6$ & $9.4 \pm 0.6(0.3)$ & $501 \pm 119$ & 481 & 524 & 557 & 581 & 595 \\
\hline 9 & $2.5 \pm 0.5$ & $8.7 \pm 0.5(0.3)$ & $490 \pm 98$ & 488 & 530 & 562 & 589 & 600 \\
\hline 10 & $2.3 \pm 0.7$ & $8.5 \pm 0.7(0.3)$ & $489 \pm 92$ & 489 & 532 & 565 & 590 & 602 \\
\hline 11 & $3.0 \pm 0.9$ & $9.8 \pm 0.9(0.2)$ & $671 \pm 115$ & 476 & 523 & 556 & 576 & 593 \\
\hline 12 & $1.4 \pm 0.4$ & $7.9 \pm 0.4(0.1)$ & $621 \pm 138$ & 492 & 541 & 575 & 593 & 608 \\
\hline 13 & $5.5 \pm 2.3$ & $13.5 \pm 2.3(0.7)$ & $613 \pm 157$ & 451 & 496 & 527 & 548 & 569 \\
\hline 14 & $1.8 \pm 0.5$ & $9.0 \pm 0.5(0.2)$ & $603 \pm 91$ & 482 & 530 & 564 & 582 & 599 \\
\hline 15 & $2.0 \pm 0.4$ & $8.9 \pm 0.4(0.2)$ & $591 \pm 60$ & 484 & 529 & 562 & 585 & 599 \\
\hline 16 & $5.1 \pm 1.7$ & $12.7 \pm 1.7(0.4)$ & $583 \pm 104$ & 457 & 499 & 530 & 554 & 573 \\
\hline 17 & $3.1 \pm 0.6$ & $8.5 \pm 0.6(0.3)$ & $561 \pm 93$ & 491 & 531 & 563 & 592 & 601 \\
\hline 18 & $6.1 \pm 2.6$ & $8.4 \pm 2.6(0.8)$ & $527 \pm 140$ & 506 & 526 & 557 & 609 & 600 \\
\hline 19 & $0.6 \pm 0.1$ & $8.3 \pm 0.1(0.04)$ & $508 \pm 42$ & 488 & 538 & 572 & 589 & 605 \\
\hline
\end{tabular}

Beside, the unbound probability from such a Monte-Carlo simulation can only represents the probability of unbound to the Galaxy when kinematic parameters are in given error ranges.

\section{METALLICITY DISTRIBUTION AND POSSIBLE ORIGINS}

The metallicity distribution investigation of Galactic populations, including the Galactic bulge, the Galactic disk, the Galactic halo, and the globular cluster, indicate each population have a significantly different metal abundance distribution from others. Meanwhile, metal abundance $([\mathrm{Fe} / \mathrm{H}])$ for a star reflects that of the place where it was born. So, metallicity distribution can be used as a tool to explore the origin of our HVS candidates.

Sadler et al. (1996) measured $[\mathrm{Fe} / \mathrm{H}]$ for $322 \mathrm{~K}$ giants of the Galactic bulge, and present the metallicity distribution function (MDF) for the Galactic bulge. They found that the mean abundance of their $\mathrm{K}$ giant samples is $\langle[\mathrm{Fe} / \mathrm{H}]>=-0.11 \pm 0.03$, and over a half of them are in the range of $-0.4<$ $[\mathrm{Fe} / \mathrm{H}]<0.3$. Schlesinger et al. (2012) derived the MDF of the Galactic disk using 24,270 G and 16,847 K dwarfs from the SDSS SEGUE, different from previous investigations, this work considered observational biases for the first time, and their $\mathrm{G}$ and $\mathrm{K}$ dwarf samples are the most complete samples in both number and volume. An et al. (2013) estimated metal abundance for individual star in SDSS Stripe 
Table 3 Probabilities that these candidates are unbound in five Galactic potential models.

\begin{tabular}{|c|c|c|c|c|c|}
\hline HVS & $\mathrm{P}_{\text {unbound }}-\mathrm{Xue} 08^{a}$ & $\mathrm{P}_{\text {unbound }}$-Pacyznski90 ${ }^{a}$ & $\mathrm{P}_{\text {unbound }}-\mathrm{Koposov}_{10}{ }^{a}$ & $\mathrm{P}_{\text {unbound }}-$ Kenyon $08^{a}$ & $\mathrm{P}_{\text {unbound }}$-Gnedin $05^{a}$ \\
\hline 1 & 0.93 & 0.84 & 0.75 & 0.86 & 0.64 \\
\hline 2 & 0.80 & 0.72 & 0.64 & 0.70 & 0.54 \\
\hline 11 & 0.87 & 0.80 & 0.75 & 0.82 & 0.67 \\
\hline 12 & 0.80 & 0.70 & 0.62 & 0.71 & 0.53 \\
\hline 13 & 0.81 & 0.73 & 0.67 & 0.75 & 0.58 \\
\hline 14 & 0.80 & 0.69 & 0.60 & 0.71 & 0.51 \\
\hline 16 & 0.77 & 0.68 & 0.61 & 0.70 & 0.51 \\
\hline 15 & 0.89 & 0.75 & 0.62 & 0.77 & $-^{b}$ \\
\hline 3 & 0.61 & 0.57 & 0.52 & - & - \\
\hline 4 & 0.64 & 0.57 & 0.52 & - & - \\
\hline 5 & 0.57 & 0.52 & - & - & - \\
\hline 6 & 0.58 & 0.52 & - & - & - \\
\hline 17 & 0.69 & 0.58 & - & - & - \\
\hline 18 & 0.53 & 0.50 & - & - & - \\
\hline 7 & 0.54 & - & - & - & - \\
\hline 8 & 0.54 & - & - & - & - \\
\hline 9 & 0.51 & - & - & - & - \\
\hline 10 & 0.50 & - & - & - & - \\
\hline 19 & 0.63 & - & - & - & - \\
\hline \multicolumn{6}{|c|}{ Notes. } \\
\hline \multicolumn{6}{|c|}{$\begin{array}{l}{ }^{a} \text { Unbound probability obtained by the Xue } 08 \text {, Pacyznski90, Koposov } 10 \text {, Kenyon } 08 \text { and Gnedin } 05 \text { potential } \\
\text { model respectively. }\end{array}$} \\
\hline
\end{tabular}

82, and presented an unbiased MDF of the Galactic halo. Harris (1997) compiled a catalogue which contains basic parameters of distances, velocities, metallicities, luminosities, colors, and dynamical parameters for 147 globular clusters in the Milky Way, and we obtained the catalog from the website http://vizier.china-vo.org/viz-bin/VizieR?-source=VII/202\&-ref=VIZ55014f467633.

We compare the metallicities of our candidates with the MDFs of the Galactic bulge (Sadler et al. 1996), the Galactic disk (Schlesinger et al. 2012), the Galactic halo (An et al. 2013) and known globular clusters Harris 1997), the MDFs for each Galactic population and our candidates are shown in Figure 3 . From this figure, we can see that the metallicity distribution of our HVS candidates is well consistent with the G and K dwarf samples in the disk, and is also roughly consistent with the low-metallicity end of the Galactic bulge. Clearly, the MDF of our candidates is completely inconsistent with that of the Galactic halo and globular clusters.

In addition, we estimate $\mathrm{Mg}$ abundances $[\mathrm{Mg} / \mathrm{Fe}]$ using a profile matching method in the region of $\mathrm{Mg}$ I b lines around $\lambda 5170 \AA$ (Li et al. 2014), and they are listed in the last column of Table 1 . The external uncertainty of this profile match method may not be as large as 0.2 dex, and the upper limit of internal uncertainty is 0.3 dex estimated by the Monte-Carlo simulation. From Table 1 we can see that the $\mathrm{Mg}$ abundances of four candidates, i.e. HVS4, HVS5, HVS8 and HVS18, are larger than 0.5. We visually inspect the spectra of the four candidates, and find that the noise seriously affect spectra quality of the $\mathrm{Mg} I$ 

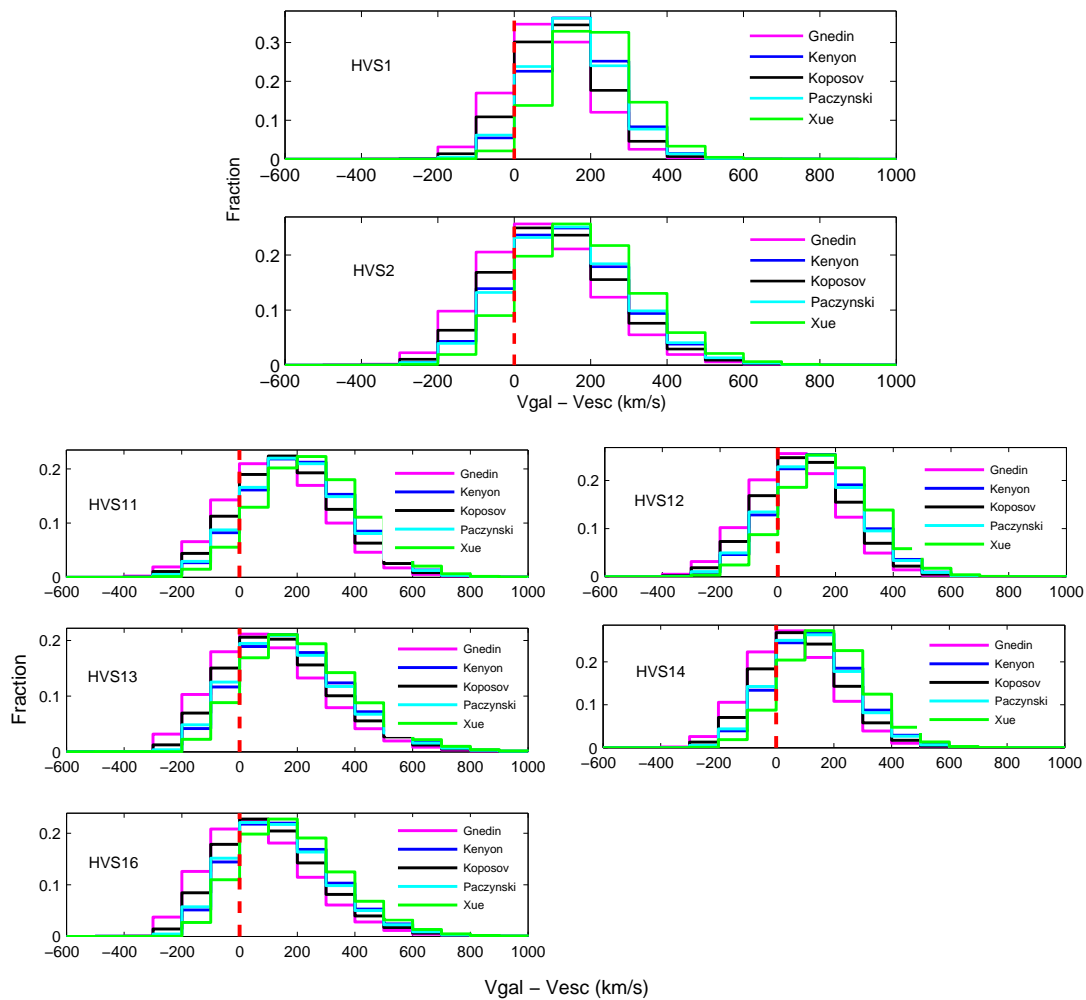

Fig. 2 The difference distribution of $V_{\text {gal }}$ and $V_{\text {esc }}$, where $V_{\text {gal }}$ and $V_{\text {esc }}$ are total Galactic velocities and escape velocities of each sample realization in a million random samples of Monte-Carlo simulation.

b region which is extremely important for the $[\mathrm{Mg} / \mathrm{Fe}]$ estimation. So, we do not regard the four candidates as Mg-enhanced stars. For other candidates, they have high quality spectra in the $\mathrm{Mg} \mathrm{I} \mathrm{b}$ region, and their $[\mathrm{Mg} / \mathrm{Fe}]$ fall within the range of error. Similarly, the $[\mathrm{Mg} / \mathrm{Fe}]$ values of our candidates are roughly consistent with the Galactic bulge and disk.

Therefore, our candidates are likely originated from the Galactic bulge and disk, and the Galactic halo and globular cluster may not be their possible origin place. However, the determination of exact birth place need much more reliable parameters, will be released in the future data release of LAMOST, to calculate the intersection regions of our candidates' trajectories and the Galactic disk.

\section{DISCUSSION AND CONCLUSIONS}

In this paper, we present $19 \mathrm{~F}, \mathrm{G}$ or $\mathrm{K}$ type hyper-velocity star candidates from over one million stars of the first data release of the LAMOST regular survey. We initially select over half a million F, G and K dwarfs with Teff and $\log g$ criteria, and then further pick out over 190,000 final F, G and K dwarf samples with a series of photometric criteria. Then, we obtain $6 \mathrm{D}$ phase space coordinates and escape velocities for each dwarf, and select 17 hyper-velocity star candidates with 'clean' proper-motions, and 15 candidates with 'reliable' proper-motions. We finally individually inspect spectra of the 32 HVS candidates, and find 19 of them have high quality spectra. Through checking with previous four low mass HVS catalogues in literatures, we conclude that they are all new findings. 

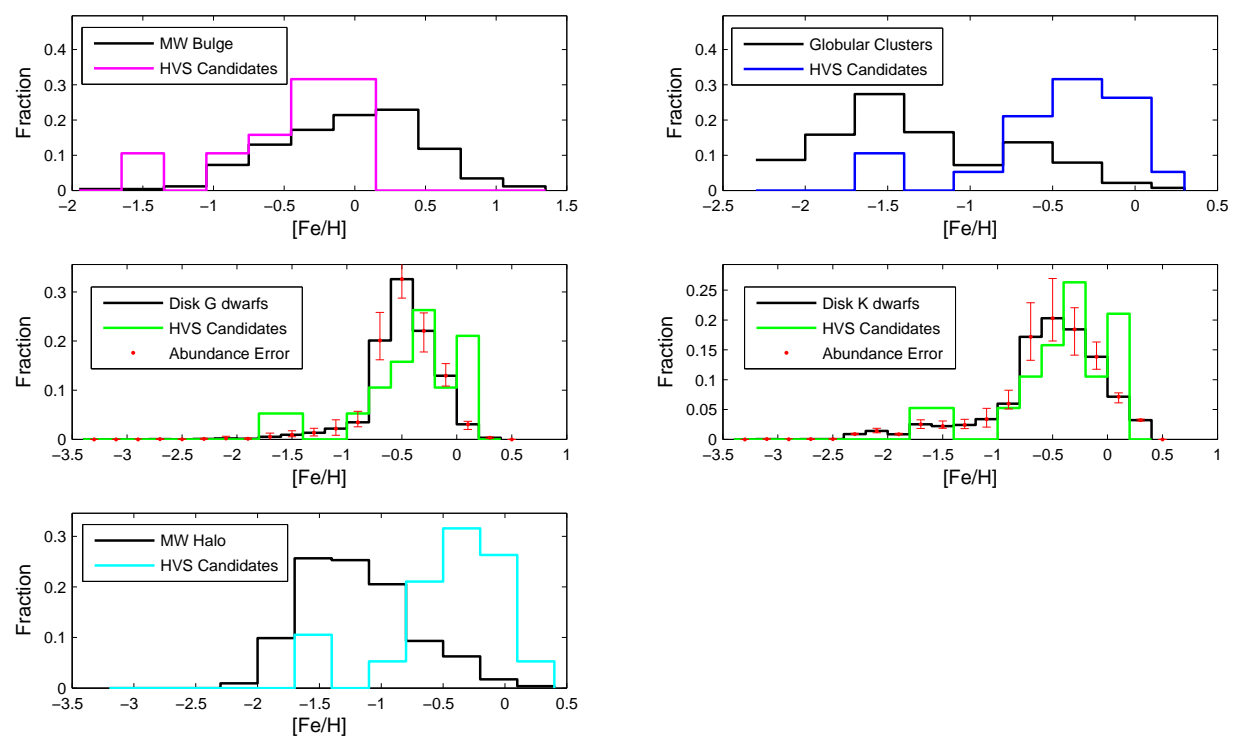

Fig. 3 The comparison of metallicity distribution of 19 HVS candidates (black solid line) with the Galactic bulge (magenta), disk (green), halo (cyan) and globular clusters (blue).

Although we use a strict criteria to ensure reliability of the kinematic parameters of our candidates, we still can not confirm that we identify 19 HVS. Therefore, we calculate the unbound probability for each candidate using the Monte-Carlo simulation, assuming a non-Gaussian proper-motion error distribution and Gaussian heliocentric distance and radial velocity error distribution. Such a probability shows each of our candidates can escape from the Milky Way in what extent. We find all the candidates have unbound probabilities over $50 \%$, one of them can even exceed escape velocity with over $90 \%$ probability, and the unbound probability varies in different potential model for each candidate. To investigate the origin of our candidates, we compare metallicities of our candidates with MDFs of the Galactic bulge, disk, halo and globular cluster, and conclude that the Galactic bulge or disk are likely the birth place of our candidates, and the Galactic halo and globular cluster seems not to be their possible origin places.

When we select HVS candidates, there are a large amount of stars with dist $22<7$, which implies that they suffer from photometry blending from a near neighborhood. There also exist a large fraction of stars with $\mathrm{nFit}<5$, which means that they have few position detections. The Hubble Space Telescope (HST) Fine Guidance Sensor (FGS) and future GAIA can provide more accurate proper-motion measurements and confirm HVS from them, and they can also help us to verify proper motions for our 19 HVS candidates. In addition, high resolution spectroscopic observations are extremely essential to obtain more accurate measurements of stellar atmospheric parameters, which may help us to determine more accurate origin places through calculating trajectories and detailed metallicity distribution analysis, and can also decide whether our HVS candidates are in binaries.

If there exist candidates in binaries, their heliocentric distances and Galactic total velocities will be systematically under-estimated, and their escape velocities will be correspondingly over-estimated, thus these binaries should more likely be able to escape from our Galaxy. Besides, we estimated the effect 
of binary orbital velocities on the observed heliocentric radial velocities and the Galactic total velocities assuming three types of companion (e.g., a solar mass main sequence companion, a neutron star companion, or a black hole companion) in our previous work (Li, et al. 2012), and we can see that average effect of binary do not exceed $100 \mathrm{~km} \mathrm{~s}^{-1}$, which have little effect on our results.

Acknowledgements We thank an anonymous referee for very useful comments that improved the presentation of the paper. We thank Palladino Lauren E. for valuable discussion of proper motion random sample in unbound probability simulation. This work is supported by the National Science Foundation of China under Grant Nos. 11303036, 11390371/4, 11233004. The Guoshoujing Telescope (the Large Sky Area Multi-Object Fiber Spectroscopic Telescope LAMOST) is a National Major Scientific Project built by the Chinese Academy of Sciences. Funding for the project has been provided by the National Development and Reform Commission. LAMOST is operated and managed by the National Astronomical Observatories, Chinese Academy of Sciences. The web site of LAMOST DR1 is http://dr1.lamost.org

\section{References}

Abadi, M. G., Navarro, J. F., \& Steinmetz, M. 2009, ApJ, 691, L63

An, D., Beers, T. C., Johnson, J. A., et al. 2013, ApJ, 763, 65

Bartko, H., Martins, F., Trippe, S., et al. 2010, ApJ, 708, 834

Blaauw, A. 1961, Bull. Astron. Inst. Netherlands, 15, 265

Bromley, B. C., Kenyon, S. J., Geller, M. J., et al. 2006, ApJ, 653, 1194

Brown, W. R., Geller, M. J., Kenyon, S. J., \& Kurtz, M. J. 2005, ApJ, 622, L33

Brown, W. R., Geller, M. J., \& Kenyon, S. J. 2009, ApJ, 690, 1639

Brown, W. R., Geller, M. J., \& Kenyon, S. J. 2012, ApJ, 751, 55

Carlin, J. L., Lépine, S., Newberg, H. J., et al. 2012, Research in Astronomy and Astrophysics, 12, 755

Chen, L., Hou, J.-L., Yu, J.-C., et al. 2012, Research in Astronomy and Astrophysics, 12, 805

Cui, X.-Q., Zhao, Y.-H., Chu, Y.-Q., et al. 2012, Research in Astronomy and Astrophysics, 12, 1197

Deng, L.-C., Newberg, H. J., Liu, C., et al. 2012, Research in Astronomy and Astrophysics, 12, 735

Dong, R., Gunn, J., Knapp, G., Rockosi, C., \& Blanton, M. 2011, AJ, 142, 116

Edelmann, H., Napiwotzki, R., Heber, U., Christlieb, N., \& Reimers, D. 2005, ApJ, 634, L181

Fellhauer, M., Belokurov, V., Evans, N. W., et al. 2006, ApJ, 651, 167

Gao, H., Zhang, H., Xiang, M., et al. 2015, inprep

Gnedin, O. Y., Gould, A., Miralda-Escudé, J., \& Zentner, A. R. 2005, ApJ, 634, 344

Geier, S., Fürst, F., Ziegerer, E., et al. 2015, Science, 347, 1126

Gunn, J. E., Carr, M., Rockosi, C., et al. 1998, AJ, 116, 3040

Gunn, J. E., Siegmund, W. A., Mannery, E. J., et al. 2006, AJ, 131, 2332

Harris, W. E. 1997, VizieR Online Data Catalog, 7202, 0

Hernquist, L. 1990, ApJ, 356, 359

Hills, J. G. 1988, Nature, 331, 687

Hirsch, H. A., Heber, U., O’Toole, S. J., \& Bresolin, F. 2005, A\&A, 444, L61

Ivezić, Ž., Sesar, B., Jurić, M., et al. 2008, ApJ, 684, 287 
Kenyon, S. J., Bromley, B. C., Geller, M. J., \& Brown, W. R. 2008, ApJ, 680, 312

Kilic, M., Munn, J. A., Harris, H. C., et al. 2006, AJ, 131, 582

Kollmeier, J. A., Gould, A., Knapp, G., \& Beers, T. C. 2009, ApJ, 697, 1543

Kollmeier, J. A., Gould, A., Rockosi, C., et al. 2010, ApJ, 723, 812

Koposov, S. E., Rix, H.-W., \& Hogg, D. W. 2010, ApJ, 712, 260

Lee, Y. S., Beers, T. C., Sivarani, T., et al. 2008, AJ, 136, 2022

Leonard, P. J. T., \& Dewey, R. J. 1993, Luminous High-Latitude Stars, 45, 239

Li, X., Zhao, G., Chen, Y.-Q., \& Li, H.-N. 2014, Research in Astronomy and Astrophysics, 14, 1423

Li, Y., Luo, A., Zhao, G., et al. 2012, ApJ, 744, LL24

Liu, X.-W., Yuan, H.-B., Huo, Z.-Y., et al. 2014, IAU Symposium, 298, 310

Lu, Y., Zhang, F., \& Yu, Q. 2010, ApJ, 709, 1356

Luo, A. L., Zhao, Y. H, Zhao, G., et al. 2015, RAA, Accepted

Luo, A.-L., Zhang, H.-T., Zhao, Y.-H., et al. 2012, Research in Astronomy and Astrophysics, 12, 1243

Merritt, D. 2006, ApJ, 648, 976

Miyamoto, M., \& Nagai, R. 1975, PASJ, 27, 533

Monet, D. G., Levine, S. E., Canzian, B., et al. 2003, AJ, 125, 984

Munn, J. A., Monet, D. G., Levine, S. E., et al. 2004, AJ, 127, 3034

Munn, J. A., Monet, D. G., Levine, S. E., et al. 2008, AJ, 136, 895

Napiwotzki, R., \& Silva, M. D. V. 2012, Mem. Soc. Astron. Italiana, 83, 272

Navarro, J. F., Frenk, C. S., \& White, S. D. M. 1996, ApJ, 462, 563

Newby, M., Newberg, H. J., Simones, J., Cole, N., \& Monaco, M. 2011, ApJ, 743, 187

O'Leary, R. M., \& Loeb, A. 2008, MNRAS, 383, 86

Paczynski, B. 1990, ApJ, 348, 485

Palladino, L. E., Schlesinger, K. J., Holley-Bockelmann, K., et al. 2014, ApJ, 780, 7

Perets, H. B. 2009, ApJ, 690, 795

Perets, H. B. 2009, ApJ, 698, 1330

Sadler, E. M., Rich, R. M., \& Terndrup, D. M. 1996, AJ, 112, 171

Schlesinger, K. J., Johnson, J. A., Rockosi, C. M., et al. 2012, ApJ, 761, 160

Schlegel, D. J., Finkbeiner, D. P., \& Davis, M. 1998, ApJ, 500, 525

Schönrich, R., Binney, J., \& Dehnen, W. 2010, MNRAS, 403, 1829

Sesana, A., Haardt, F., \& Madau, P. 2007, MNRAS, 379, L45

Su, D.-Q., \& Cui, X.-Q. 2004, ChJAA (Chin. J. Astron. Astrophys.), 4, 1

Teyssier, M., Johnston, K. V., \& Shara, M. M. 2009, ApJ, 707, L22

Wang, S.-G., Su, D.-Q., Chu, Y.-Q., Cui, X., \& Wang, Y.-N. 1996, Appl. Opt., 35, 5155

Wang, B., \& Han, Z. 2009, A\&A, 508, L27

Xiang, M. S., Liu, X. W., Yuan, H. B., et al. 2015, MNRAS, 448, 822

Xue, X. X., Rix, H. W., Zhao, G., et al. 2008, ApJ, 684, 1143

Yang, F., Carlin, J. L., Liu, C., et al. 2012, Research in Astronomy and Astrophysics, 12, 781

Yanny, B., Rockosi, C., Newberg, H. J., et al. 2009, AJ, 137, 4377 
York, D. G., Adelman, J., Anderson, J. E., Jr., et al. 2000, AJ, 120, 1579

Yu, Q., \& Tremaine, S. 2003, ApJ, 599, 1129

Yuan, H. B., Liu, X. W, Huo, Z. Y, et al. 2014, ArXiv e-prints

Zhang, F., Lu, Y., \& Yu, Q. 2010, ApJ, 722, 1744

Zhang, Y.-Y., Carlin, J. L., Yang, F., et al. 2012, Research in Astronomy and Astrophysics, 12, 792

Zhang, F., Lu, Y., \& Yu, Q. 2013, ApJ, 768, 153

Zhao, G., Zhao, Y.-H., Chu, Y.-Q., Jing, Y.-P., \& Deng, L.-C. 2012, Research in Astronomy and Astrophysics, 12, 723

Zheng, Z., Carlin, J. L., Beers, T. C., et al. 2014, ApJ, 785, LL23

Zhong, J., Chen, L., Liu, C., et al. 2014, ApJ, 789, L2 